immunosuppressive drugs. At the time of data cut-off, all subjects who entered OLE completed 12 months of dosing.

Nineteen/20 (95\%) of subjects experienced at least $1 \mathrm{AE}$, with $59 \mathrm{AEs}$ occurring among the subjects during the OLE to date. The majority of AEs were mild $(n=16$, $80 \%$ ), with 1 severe $A E$ (fatigue) considered unrelated to lenabasum reported. AEs occurring in $\geq 2$ subjects were: dermatomyositis worsening, dizziness, fatigue, herpes zoster, nasopharyngitis, nausea, upper respiratory tract infection, and urinary tract infection. No serious AEs related to lenabasum have been reported.

Improvement was seen in multiple physician- and patient-reported efficacy outcomes; selected outcomes are presented in Figure 1. Mean (SE) changes from study start at Week 52 in the OLE were: CDASI activity score $=-17.6$ (SD), Patient Skin Activity VAS = -2.6 (SD); Skindex-29 Symptoms Domain = -21.6 (SD); Patient Itch VAS = -2.8 (SD); Physician Overall Disease VAS = -3.0 (SD); and Patient Pain $\mathrm{VAS}=-2.3(\mathrm{SD})$. Improvements were seen in other efficacy outcomes. 12 subjects had no changes in immunosuppressive drugs during the OLE, 3 reduced chronic steroids, 2 reduced mycophenolate, 3 were switched from methotrexate to mycophenolate, 1 started methotrexate, and 1 had a burst and taper of steroids.
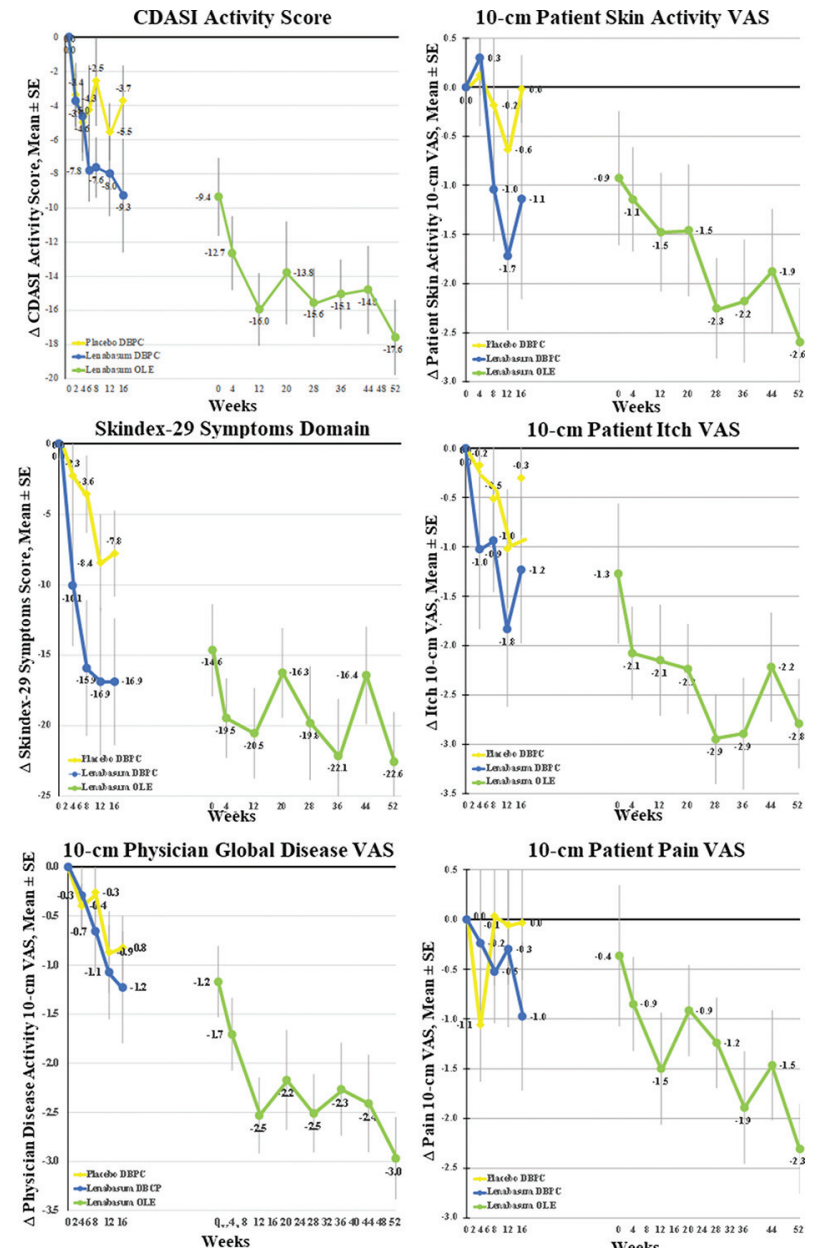

Weeks

Figure 1. Change from Baseline in Selected Efficacy Outcomes in OLE of Phase 2 Trial JBT101-DM-001

Conclusion: : Lenabasum continues to have a favorable safety and tolerability profile in the OLE of the Phase 2 trial JBT101-DM-001 with no serious AEs or study discontinuations related to lenabasum. The CDASI activity score and multiple other physician and patient-reported outcomes improved, although limitations of attributing efficacy to lenabasum in the setting of open-label dosing is acknowledged. These data support further testing of lenabasum for the treatment of DM, and a Phase 3 study of lenabasum in DM has started.

Disclosure of Interests: : Victoria Werth: None declared, David Pearson: None declared, Joyce Owaka: None declared, Rui Feng: None declared, Josef Simon Concha: None declared, Basil Patel: None declared, Emily Hejazi: None declared, Caitlin Cornwall Shareholder of: Corbus Pharmaceuticals, Inc., Employee of: Corbus Pharmaceuticals, Inc., Scott Constantine Shareholder of: Corbus Pharmaceuticals, Inc., Employee of: Corbus Pharmaceuticals, Inc., Nancy Dgetluck Employee of: Corbus Pharmaceuticals, Inc., Barbara White Shareholder of: Corbus Pharmaceuticals, Inc., Employee of: Corbus Pharmaceuticals, Inc.
DOI: 10.1136/annrheumdis-2019-eular.3397

\section{OP0242 \\ SAFETY PROFILE OF NINTEDANIB IN PATIENTS WITH SYSTEMIC SCLEROSIS-ASSOCIATED INTERSTITIAL LUNG DISEASE AND IDIOPATHIC PULMONARY FIBROSIS}

Kristin Highland ${ }^{\star 1}$, Oliver Distler ${ }^{2}$, Martina Gahlemann ${ }^{3}$, Arata Azuma ${ }^{4}$, Aryeh Fischer ${ }^{5}$, Maureen Mayes ${ }^{6}$, Ganesh Raghu' ${ }^{7}$, Wiebke Sauter ${ }^{8}$, Mannaig Girard ${ }^{9}$, Veronika Kohlbrenner ${ }^{10}$, Emmanuelle Clerisme-Beaty ${ }^{11}$, Susanne Stowasser ${ }^{11}$, Masataka Kuwana ${ }^{12}$, Toby M Maher $^{13}$, SENSCIS trial investigators. ${ }^{1}$ Respiratory Institute, Cleveland Clinic, Cleveland, Ohio, United States of America; ${ }^{2}$ Dept of Rheumatology, University Hospital Zurich, Zurich, Switzerland; ${ }^{3}$ Boehringer Ingelheim (Schweiz) GmbH, Basel, Switzerland; ${ }^{4}$ Dept of Pulmonary Medicine and Oncology, Graduate School of Medicine, Nippon Medical School, Tokyo, Japan; ${ }^{5}$ University of Colorado School of Medicine, Denver, Colorado, United States of America; ${ }^{6}$ Division of Rheumatology and Clinical Immunogenetics, University of Texas McGovern Medical School, Houston, Texas, United States of America; ${ }^{7}$ University of Washington, Seattle, United States of America; ${ }^{8}$ Boehringer Ingelheim Pharma GmbH and Co. KG, Biberach an der Riss, Germany, ${ }^{9}$ Boehringer Ingelheim France S.A.S., Reims, France; ${ }^{10}$ Boehringer Ingelheim Pharmaceuticals, Inc., Ridgefield, Connecticut, United States of America; ${ }^{11}$ Boehringer Ingelheim International $\mathrm{GmbH}$, Ingelheim am Rhein, Germany, ${ }^{12}$ Dept of Allergy and Rheumatology, Nippon Medical School Graduate School of Medicine, Tokyo, Japan; ${ }^{13}$ National Heart and Lung Institute, Imperial College London, UK, and National Institute for Health Research Clinical Research Facility, Royal Brompton Hospital, London, United Kingdom

Background: Nintedanib has been investigated in patients with systemic sclero sis-associated interstitial lung disease (SSc-ILD) in the SENSCIS trial and idiopathic pulmonary fibrosis (IPF) in the two INPULSIS trials. These patient populations differ in age, sex, disease characteristics and comorbidities.

Objectives: To compare the safety and tolerability of nintedanib in patients with SSc-ILD and IPF

Methods: Adverse events that occurred over 52 weeks of treatment in the SENS CIS and INPULSIS trials were assessed descriptively in subjects who received $>1$ dose of trial drug.

Results: A total of 576 subjects were treated in the SENSCIS trial (288 nintedanib; 288 placebo) and 1061 in the INPULSIS trials (638 nintedanib; 423 placebo). At baseline, mean (SD) age was 54.0 (12.2) and 66.8 (8.0) years in SENSCIS and INPULSIS, respectively. The proportion of males was $24.8 \%$ and $79.3 \%$, respectively. Over 52 weeks, $19.4 \%$ and $10.8 \%$ of patients treated with nintedanib and placebo discontinued treatment in SENSCIS, compared with $24.5 \%$ and $18.9 \%$ of patients treated with nintedanib and placebo in INPULSIS. Gastrointestinal adverse events were the most frequently reported adverse events with nintedanib and, as expected based on the underlying disease, were more frequent in patients with SSc-ILD than IPF in both treatment groups (Table). Diarrhoea adverse events were reported in $75.7 \%$ and $31.6 \%$ of patients treated with nintedanib and placebo in SENSCIS, and $62.4 \%$ and $18.4 \%$ of patients treated with nintedanib and placebo in INPULSIS, respectively.

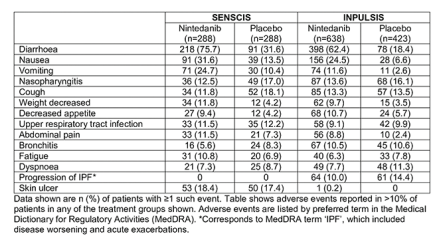

Conclusion: The safety and tolerability profile of nintedanib in patients with SSc ILD is similar to that observed in patients with IPF.

Disclosure of Interests: DOI: 10.1136/annrheumdis-2019-eular.3237

\section{OP0243 EFFICACY OF PIRFENIDONE IN SYSTEMIC SCLEROSIS RELATED INTERSTITIAL LUNG DISEASE - A RANDOMISED CONTROLLED TRIAL}

Nupoor Acharya ${ }^{1}$, Debasish Mishra ${ }^{1}$, Sahajal Dhooria ${ }^{2}$, Varun Dhir $^{1}$, Sanjay Jain ${ }^{1}$, Shefali Khanna Sharma'. ${ }^{1}$ Post Graduate Institute of Medical Education and Research, Chandigarh, Internal medicine, Division of Rheumatology, Chandigarh, India; ${ }^{2}$ Post Graduate Institute of Medical Education and Research, Chandigarh, Pulmonary Medicine, Chandigarh, India

Background: Interstitial lung disease (ILD) is a major cause of morbidity and mortality in systemic sclerosis(SSc).(1) Lung fibrosis in systemic sclerosis shares 